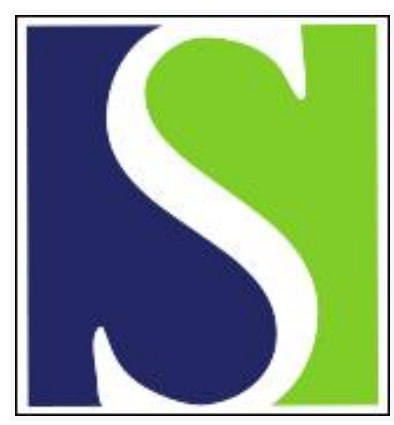

Scand J Work Environ Health 1999;25(1):18-23

https://doi.org/10.5271/sjweh.378

Issue date: Feb 1999

Magnetic resonance appearance of asbestos-related benign and malignant pleural diseases

by Boraschi P, Neri S, Braccini G, Gigoni R, Leoncini B, Perri G

Key terms: magnetic resonance; mesothelioma; plaque; pleura; pleural mesothelioma; pleural plaque; preliminary study; tissue characterization

This article in PubMed: www.ncbi.nlm.nih.gov/pubmed/10204666

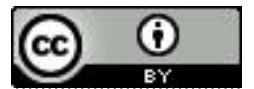




\title{
Magnetic resonance appearance of asbestos-related benign and malignant pleural diseases
}

\author{
by Piero Boraschi, MD, ${ }^{1}$ Silvano Neri, MD, ${ }^{2}$ Giovanni Braccini, MD, ${ }^{1}$ Roberto Gigoni, MD, ${ }^{1}$ \\ Benito Leoncini, MD, ${ }^{3}$ Giuseppe Perri, $M D^{1}$
}

Boraschi P, Neri S, Braccini G, Gigoni R, Leoncini B, Perri G. Magnetic resonance appearance of asbestos-related benign and malignant pleural diseases. Scand J Work Environ Health 1999;25(1):18—23.

\begin{abstract}
Objectives This study describes the magnetic resonance findings of benign and malignant pleural diseases in asbestos-exposed subjects.

Methods Thirty patients with a history of asbestos exposure and pleural lesions in chest X-rays and computed tomography scans were examined with a 0.5 - and a 1.5-T magnetic resonance unit. The examination protocol included cardiac-gated proton density and T2-weighted images, unenhanced and enhanced (Gd-DTPA; $0.1 \mathrm{mmol} /$ kg) T1-weighted images in the axial plane and sometimes in another orthogonal plane (sagittal or coronal or both). All the magnetic resonance images were reviewed by 3 experienced observers, who visually evaluated morphologic features, signal intensity, and contrast enhancement of pleural lesions. The diagnosis was established by means of percutaneous biopsy, thoracotomy, and combined clinical and radiological follow-up for at least 3 years.

Results Eighteen patients affected with multiple pleural plaques showed low signal intensity on both unenhanced and enhanced T1-weighted and proton density and T2-weighted images. In 2 of these patients an acute pleural effusion was observed. All the malignant lesions (11 mesotheliomas) and a solitary benign pleural plaque revealed high signal intensity on the proton density and T2-weighted images and inhomogeneous contrast enhancement in the postcontrast T1-weighted images. The sensitivity, specificity, and diagnostic accuracy of the magnetic resonance imaging in classifying a lesion as suggestive of malignancy were $100 \%, 95 \%$ and $97 \%$, respectively.

Conclusions The results point out 2 magnetic resonance signal intensity patterns for asbestos-related pleural lesions: (i) low-signal intensity on unenhanced and enhanced T1-weighted and proton density and T2-weighted images for benign plaques and (ii) nonhomogeneous hyperintensity in T2-weighted and enhanced T1-weighted images for malignant mesotheliomas.
\end{abstract}

Key terms magnetic resonance, tissue characterization; pleura; pleura, mesothelioma; pleura, plaques; preliminary study.

Pleural plaques are the most common, and often the only detectable disease among workers exposed to asbestos (1, 2). The prevalence of pleural plaques correlates strongly with the length of exposure and the latency time since the first exposure (2-4), and it depends, obviously, on the type of technique used to detect the lesions. In epidemiologic studies based on chest X-ray examinations, the occurrence of pleural plaques has been estimated to be $14 \%$ to $62 \%$ among heavily asbestos-exposed workers and $2 \%$ to $17 \%$ among persons with environmental exposure (5-8). By means of autopsy, the occurrence of pleural plaques among persons with "definite" exposure to asbestos has been shown to be up to $73 \%$ (9). Further studies, based on high-resolution computed tomography (HRCT) $(4,10)$, showed a prevalence of pleural plaques similar to that of pathological studies, and they also confirmed what Jarvholm hypothesized already in the mid 1980s: ". . . it is probable that almost all

1 2nd Department of Radiology, Pisa University Hospital, Pisa, Italy.

2 National Health Service - ASL 1 Tuscany, Unit of Preventive Medicine, Carrara, Italy.

3 Department of Pneumology, Pisa University Hospital, Pisa, Italy.

Reprint requests to: Dr Piero Boraschi, 2nd Department of Radiology, Pisa University Hospital, Via Paradisa 2, I-56124 Pisa, Italy. [E-mail: p.boraschi@do.med.unipi.it] 
persons with heavy exposure to asbestos have pleural plaques if they survive longer than the required number of years" [p 32] (3).

Tumors of the pleura are classified as either diffuse or localized. Diffuse malignant mesothelioma, the most common primary tumor of the pleura, is associated with a poor prognosis $(1,11,12)$ and is generally related to asbestos exposure. In contrast, localized fibrous tumors are rare, generally associated with a good prognosis after surgery, and usually not related to asbestos. Recently, it has been shown that computed tomography (CT) may play a major role in distinguishing malignant from benign pleural diseases, even if malignant mesothelioma could not be reliably differentiated from pleural metastases $(13,14)$. Leung et al (13) concluded that helpful CT features in distinguishing malignant from benign pleural diseases are pleural rind, nodular pleural thickening, parietal pleural thickening greater than $1 \mathrm{~cm}$, and mediastinal pleural involvement.

The role of magnetic resonance imaging (MRI) in the evaluation of pleural abnormalities is somewhat limited $(15,16)$ and, to our knowledge, only a few reports have described the MRI features of asbestos-related benign and malignant pleural diseases $(12,17-20)$.

In this report we present our experience with a group of asbestos-exposed patients affected with pleural diseases to evaluate whether MRI would be useful in differentiating benign from malignant lesions.

\section{Subjects and methods}

Thirty patients ( 26 men and 4 women), aged $45-85$ (mean 58.0, SD 11.5) years and seen consecutively were referred to our institution for an MRI examination of the chest because of suspected malignancy of pleural lesions identified by CT. All the patients had a history of exposure to asbestos and were previously examined with chest radiographs and CT scans.

Magnetic resonance (MR) images were acquired with a 0.5-T MR system for 26 patients (MR Max Plus and Contour, GE Medical System, Milwaukee, WI, USA) and with a 1.5-T unit for 4 patients (Signa, GE Medical System, Milwaukee, WI, USA).

All the MRI examinations were performed with the use of the conventional Spin-Echo (SE) technique and a body coil for both excitation and signal reception.

Cardiac-gated T1-weighted images (450-600/2030 milliseconds TR/TE, 4 NEX) were obtained before and after an intravenous injection of a bolus of $0.1 \mathrm{mmol} /$ kg gadopentetate dimeglumine (Magnevist ${ }^{\circledR}$, Schering, Berlin, Germany). The MRI examination protocol also included cardiac-gated proton density and T2-weighted images (1800-2200/40-120 milliseconds TR/TE, 2 NEX). The slice thickness was $10 \mathrm{~mm}$, a matrix of
$224 \times 160$ was used, the field of view ranged between 38 and $48 \mathrm{~cm}$, and all the lesions were imaged in the axial plane and sometimes in an orthogonal plane (sagittal or coronal or both).

All the MR images were analyzed by 3 experienced radiologists, who were also familiar with other imaging techniques, but they did not have information about the results of the $\mathrm{CT}$ scan or the chest radiograph or about the patients' history when they assessed the images. They interpreted the images in conference and reached a final decision by consensus.

The observers subjectively compared the signal intensity of the pleural abnormalities on T1-weighted, proton density-weighted, T2-weighted, and contrast-enhanced T1-weighted images with that of the muscle of the chest wall.

They were asked to assess whether the signal intensity of the pleural lesions was isointense, hypointense, or hyperintense in comparison with that of the muscle for each pulse sequence.

Furthermore, the reviewers reported their confidence in diagnosing benignity versus malignancy for the pleural lesions on the basis of morphologic features (thickness of pleural lesion, regularity of internal and external margins, mediastinic pleural involvement, circumferential pleural thickening or "pleural rind", pleurisy, and diffusion to other structures), structure, and signal intensity in T1- and T2-weighted SE images.

A pleural lesion thickness of $\leq 1 \mathrm{~cm}$, regular margins, hypo- or isointense signal intensity relative to that of muscle in T2-weighted images, and homogeneous lowcontrast enhancement were considered as criteria for the benignity of the lesions. On the contrary, the criteria for malignancy were thickness of pleural lesion $>1 \mathrm{~cm}$, irregular margins, mediastinic pleural involvement, presence of "pleural rind", pleurisy, diffusion to other structures of the chest, and inhomogeneous hyperintense signal intensity on T2-weighted images and on enhanced T1-weighted images.

The final diagnosis of malignancy (malignant pleural mesotheliomas) was established by means of percutaneous biopsy ( 7 patients) and thoracotomy (4 patients). The benign diseases (asbestos-related pleural plaques) were diagnosed with percutaneous biopsy (1 patient) or combined clinical and radiological (chest X-ray or $\mathrm{CT}$ or both) follow-up for at least 3 years (18 patients).

As a result of the observations, the cases in which MRI correctly identified a malignancy of pleural lesions were defined as true positive; false positives were the cases in which the detection of malignancy was not confirmed by percutaneous biopsy, thoracotomy, or clinical and radiological follow-up; false negatives were the cases with no evidence of malignancy in the MRI examination but for which malignancy was detected by means of percutaneous biopsy, thoracotomy, or clinical and 
radiological follow-up; and true negatives were those for which there was no detection of malignancy in the final diagnosis.

The sensitivity, specificity, global diagnostic accuracy, positive predictive value (PPV), and negative predictive value (NPV) of the MR findings were determined.

\section{Results}

All malignant mesotheliomas showed a low-to-intermediate signal intensity in the T1-weighted images and an inhomogeneous high signal intensity both in the proton density and T2-weighted and in the contrast-enhanced T1-weighted images (figure 1).

The thickness of the pleural lesions was greater than $1 \mathrm{~cm}$ and the internal margins were irregular in all the patients. Of the 11 cases, irregular external margins were found in 9, mediastinic pleural involvement in 8 , "pleural rind" in 7, pleurisy in 7, and diffusion to other mediastinic structures in 7 .

On the other hand, in 18 of the 19 patients affected with benign lesions, the signal intensity of the pleural plaques was low both in the T1-weighted and in the proton density and T2-weighted images (figure 2); in 2 of
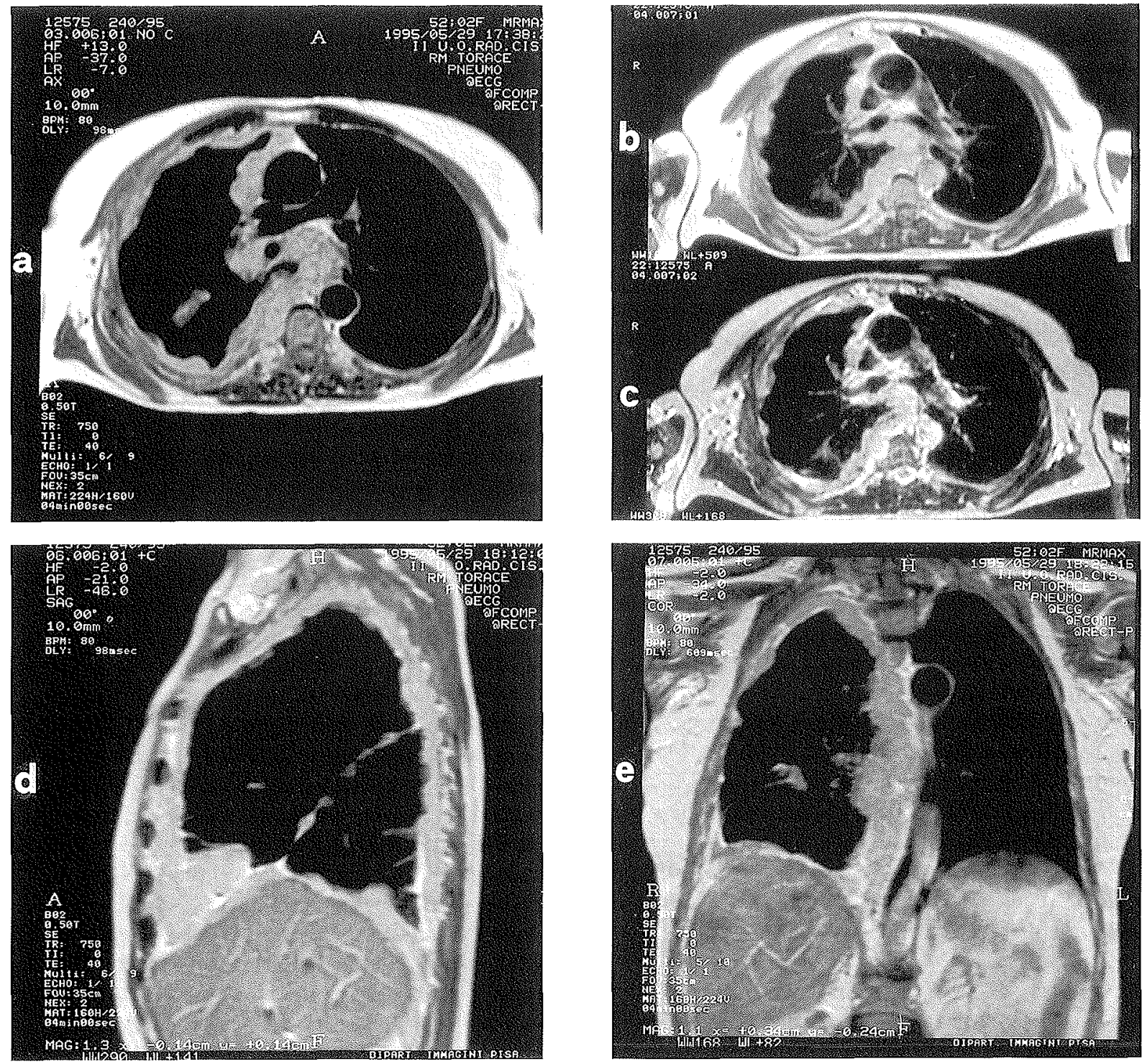

Figure 1. Malignant diffuse pleural mesothelioma. The axial T1-weighted (a) magnetic resonance image shows a right circumferential pleural thickening ("pleural rind") with irregular margins and intermediate signal intensity. In the proton density (b) and T2-weighted (c) sequences the structure of the tumor is heterogeneous, of high signal intensity. Enhanced Tt-weighted images in the sagittal (d) and coronal (e) planes demonstrate the contrast enhancement of "pleural rind" and tumor extending along the diaphragm and descending aorta. Fissural involvement can be seen. 

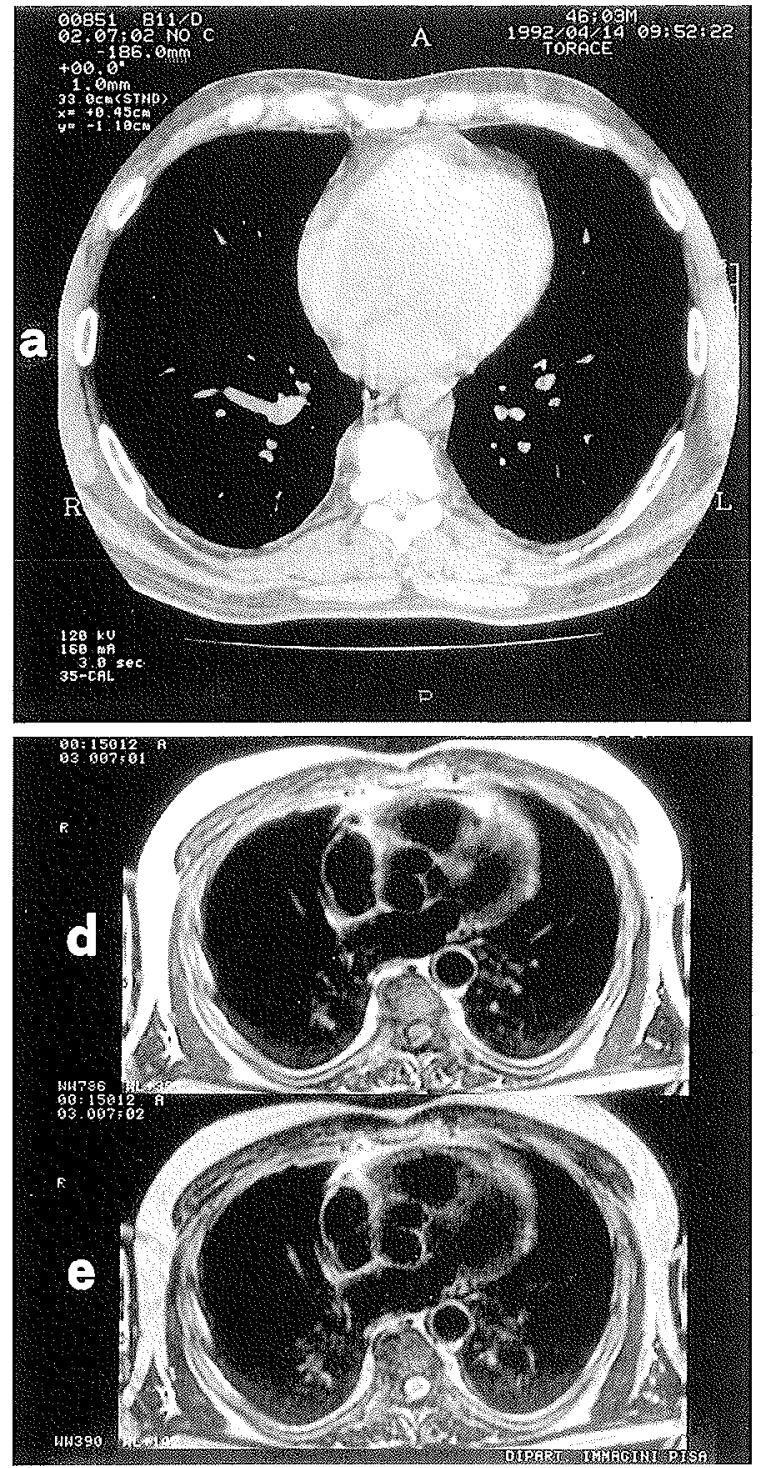

these patients a pleural effusion was identified (figure 3 ). In 1 patient a benign pleural plaque was hyperintense both in the proton density and the T2-weighted images. After the intravenous administration of gadolinium, 4 benign lesions showed contrast enhancement, 2 of them with inhomogeneous structure.

The thickness of pleural plaques was $\leq 1 \mathrm{~cm}$ in 15 out of 19 patients, and the internal margins were regular in 14 of 19 persons. In no patient was an irregularity of external margins, "pleural rind", mediastinic pleural involvement, or diffusion to other mediastinic structures identified.

Table 1 presents the statistical measures of the 3 observers in the identification of pleural malignancy.

Overall, on the basis of the readings of the 3 radiologists, the MRI had a sensitivity of $100 \%$, a specificity of $95 \%$, a positive predictive value of $92 \%$, a negative predictive value of $100 \%$, and a diagnostic accuracy of $97 \%$ in the detection of pleural malignancy.

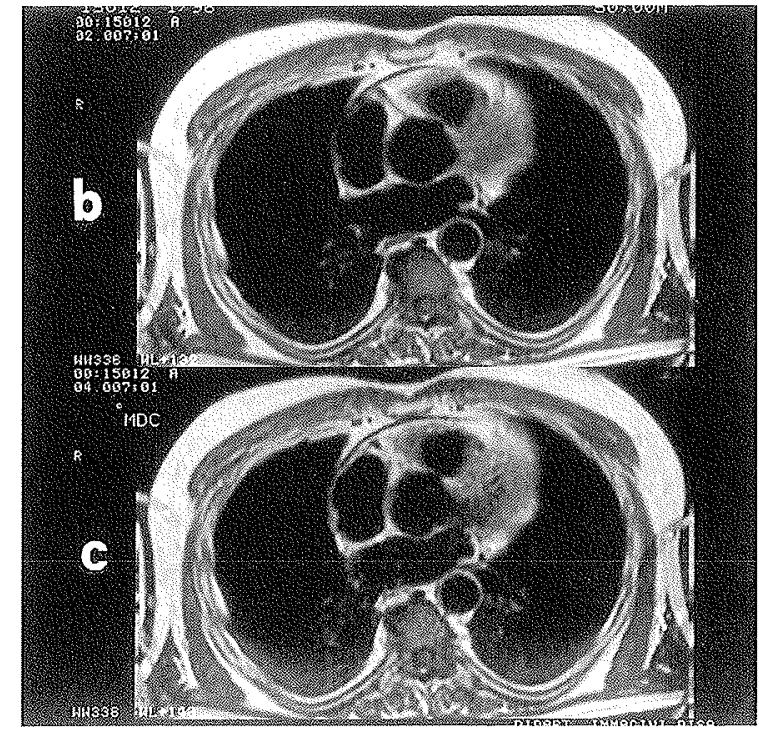

Figure 2. Asbestos-related benign pleural plaques. A high-resolution computed tomographic scan (a), photographed at a "mediastinal window", demonstrates the presence of thin and circumscribed pleural thickenings with regular margins at the level of the costovertebral spaces. Both axial unenhanced (b) and enhanced (c) T1-weighted and axial proton density (d) and T2-weighted (e) magnetic resonance images show a low signal intensity of thin bilateral pleural plaques.

Finally, 5 of the investigated subjects had asbestosis, and the asbestosis did not limit the diagnostic usefulness of the MRI.

\section{Discussion}

In most cases of pleural disease, the clinical issues necessary for management can be evaluated by chest radiography, CT, or ultrasonosgraphy $(15,16)$

MRI currently plays a very limited role in the assessment of pulmonary and pleural diseases, although it seems to be the imaging of choice in the evaluation of superior sulcus carcinoma $(21,22)$ and may provide an effective noninvasive means for the initial characterization and serial follow-up of such pleural fluid collections as transudate, exudate, or complex (23). The high signal intensity of lipids in T1-weighted sequences allows the identification of chylothorax (15). 

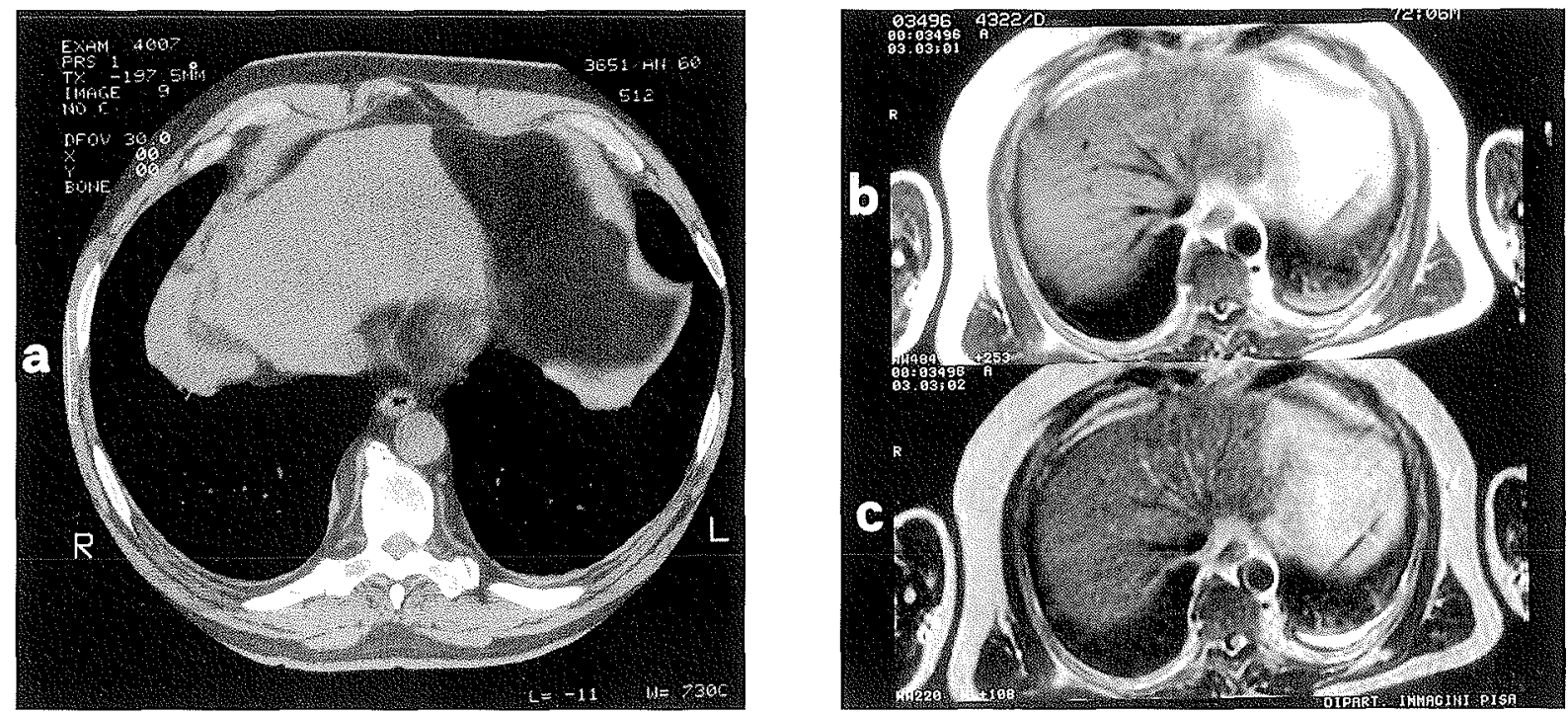

Figure 3. Asbestos-related pleural plaques with a left benign pleural effusion. A high-resolution computed tomographic scan (a), photographed at a "mediastinal window", reveals a bilateral and thin pleural thickening in the costovertebral space. Bilateral pleural plaques appear to be hypointense in the axial proton density (b) and T2-weighted (c) magnetic resonance images, obtained 1 year after the computed tomographic scans. A concomitant pleural effusion is evident on the left side.

Table 1. Diagnostic value of the magnetic resonance imaging for patients with benign and malignant asbestos-related pleural disease.

\begin{tabular}{lc}
\hline Test parameters & Reviewers \\
\hline Findings & \\
True-positive & 11 \\
True-negative & 18 \\
False-positive & 1 \\
False-negative & - \\
Statistical measures & \\
Sensitivity (\%) & 100 \\
Specificity (\%) & 95 \\
Accuracy (\%) & 97 \\
Positive predictive value (\%) & 92 \\
Negative predictive value (\%) & 100 \\
\hline
\end{tabular}

a Standards of reference used for the final diagnosis are given in the text.

Furthermore, MRI is superior to CT for the characterization of tissues on the basis of signal intensity in various pulse sequences, and the multiplanar capability of MRI may be useful in demonstrating the extent of pleural disease, mediastinal invasion, and chest wall invasion before surgery.

MRI findings have been described for 3 patients affected with malignant mesothelioma. The extent of the tumor was better shown by MRI than by means of CT, particularly in the coronal planes (17). In addition, the comparison of the MR images with CT scans for 16 patients diagnosed with pleural mesothelioma demonstrated that, compared with CT, MRI in the coronal plane allowed a better evaluation of apical disease, diaphragmatic and infradiaphragmatic involvement, and the relationship of tumor to mediastinal structures (12). These results are preliminary, but they do demonstrate a potential role of MRI in the assessment of malignant mesothelioma.

Ferretti et al (24) reported the MRI appearance of localized benign fibrous tumors of the pleura in 4 patients. These lesions were characterized by a low signal intensity in all the MRI sequences; this low signal intensity can be explained by high collagen content within the stroma of the tumor.

In a recent study of 55 patients with different pleural lesions, Falaschi et al (19) reported that the presence of a high signal intensity in the proton-density-weighted and T2-weighted images had a $100 \%$ sensitivity and an $87 \%$ specificity for the identification of malignant pleural diseases such as mesothelioma, metastasis, and nonHodgkin's lymphoma. Moreover, a lesion with a low signal intensity in the T2-weighted images was invariably benign (negative predictive value, 100\%).

To evaluate whether MRI could be useful in differentiating benign from malignant lesions, we selected a group of patients who had been occupationally exposed to asbestos and who had had pleural lesions in CT scans.

The following two MRI signal intensity patterns were identified for pleural lesions: (i) a low signal intensity on both unenhanced and enhanced T1-weighted and proton-density and T2-weighted images for benign pleural plaques and (ii) inhomogeneous hyperintensity in protondensity-weighted, T2-weighted images and in contrastenhanced T1-weighted images for malignant mesotheliomas.

Moreover, the observers obtained a $100 \%$ sensitivity and a $95 \%$ specificity for the identification of pleural malignancy and a $100 \%$ negative predictive value. These results suggest that MRI and, particularly, T2-weighted 
sequences may differentiate between benign and malignant pleural nodules in asbestos-exposed workers with high sensitivity and specificity.

On the other hand, some limitations were also revealed by our study.

Even if no morphological feature by itself could allow for a differentiation between malignant and benign pleural diseases, the overall evaluation of morphological signs was very helpful when a pleural lesion was being characterized. Another important limitation to our study was the relatively small number of malignant lesions included in our series. This small number limits the confidence with which one can apply our conclusions in a clinical setting.

In summary, this report emphasizes the distinctive MRI pattern of benign and malignant asbestos-related pleural diseases. Although this MRI pattern is not always pathognomonic, its presence within a pleural lesion can suggest a presumptive diagnosis and may obviate the need for percutaneous biopsy, which is often not definitive. Therefore, MRI appears to be a promising tool in the assessment of asbestos-related benign and malignant pleural diseases.

\section{References}

1. Mossman BT, Gee JBL. Asbestos-related diseases. N Engl J Med 1989;320:1721-30.

2. Selikoff IJ, Lilis R, Levin G. Asbestotic radiological abnormalities among United States merchant marine seamen. $\mathrm{Br}$ J Ind Med 1990;47:292-7.

3. Järvholm B, Arvidsson H, Bake B, Hillerdal G, Westrin CG. Pleural plaques-asbestos-ill hearth. Eur J Respir Dis 1986; 68 suppl 145:1-59.

4. Neri S, Antonelli A, Falaschi F, Boraschi P, Baschieri L. Findings from high resolution computed tomography of the lung and pleura of symptom free workers exposed to amosite who had normal chest radiographs and pulmonary function tests. Occup Environ Med 1994;51:239—43.

5. Sandén A, Larsson S, Lavenius B. Asbestexponerade varvsarbetare-en tvärsnitts-studie. Läkartidningen 1984;81:195962.

6. Hillerdal G. Pleural plaques: occurrence, exposure to asbestos and clinical importance [dissertation]. Uppsala (Sweden): Uppsala University, 1980.

7. Schwartz DA, Fuortes LJ, Galvin JR, Burmeister LF, Schmidt LE, Lestikow BN, et al. Asbestos-induced pleural fibrosis and impaired lung function. Am Rev Respir Dis 1990;141:321-

8. Schwartz DA. New developments in asbestos-related pleural disease. Chest 1991;99:191 - 8.

9. Mollo F, Andrion A, Pira E, Baroncelli M. Indicators of asbestos exposure in autopsy routine: 2 . pleural plaques and occupation. Med Lav 1983;74:137-42.

10. Falaschi F, Boraschi P, Neri S, Antonelli A, Rizzini D, Battolla L. High-resolution computed tomography (HRCT) in the detection of "early asbestosis". Eur Radiol 1995;5:291 -6.

11. Legha SS, Muggia F. Pleural mesothelioma: clinical features and therapeutic implications. Ann Intern Med 1977;87:61321.

12. Patz EF Jr, Shaffer K, Piwnica-Worms DR, Jochelson M, Sarin M, Sugarbaker DJ, et al. Malignant pleural mesothelioma: value of $C T$ and MR imaging in predicting resectability. Am J Roentgenol 1992;159:961-6.

13. Leung AN, Muller NL, Miller RR. CT in differential diagnosis of diffuse pleural disease. Am J Roentgenol 1990;154:487-92.

14. Falaschi F, Boraschi P, Musante F, Volpini F, D'Alessandro F, Torri $T$, et al. Computed tomographic diagnosis of malignant pleural mesothelioma: a multicentric study. Radiol Med 1992;84:43-7.

15. McLoud TC, Flower CDR. Imaging the pleura: sonography, CT, and MR imaging. Am J Roentgenol 1991;156:1145-53.

16. Muller NL. Imaging of the pleura. Radiology 1993;186:297 309.

17. Lorigan JG, Libshitz HI. MR imaging of malignant pleural mesothelioma. J Comput Assist Tomogr 1989;13:617-20.

18. Montalvo BM, Morillo G, Sridhar K, Christoph C. MR imaging of malignant pleural mesothelioma. Radiology 1991;181(P); 109 .

19. Falaschi F, Battolla L, Mascalchi M, Cioni R, Zampa V, Lencioni $R$, et al. Usefulness of MR signal intensity in distinguishing benign from malignant pleural disease. Am J Roentgenol 1996;166:963-8.

20. Boraschi P, Neri S, Braccini G, Cartei F, Gigoni R, Leoncini $B$, et a1. MRI features of asbestos-related pleural disease. Eur Radiol 1997;7(P) suppl 1:357.

21. McLoud TC, Filion RB, Edelman RR, Shepard JO. MR imaging of superior sulcus carcinoma. J Comput Assist Tomogr 1989;13:233-9.

22. Takasugi J, Rapoport $S$, Shaw C. Superior sulcus tumors: the role of imaging. J Thorac Imaging 1989;4:41-8.

23. Davis SD, Henschke CI, Yankelevitz DF, Cahill PT, Yi Y. MR imaging of pleural effusions. J Comput Assist Tomogr $1990 ; 14: 192-8$

24. Ferretti GR, Chiles C, Cox JE, Choplin RH, Coulomb M Localized benign fibrous tumors of the pleura: MR appearance. J Comput Assist Tomogr 1997;21:115-20.

Received for publication: 2 July 1998 